\title{
Interglaziale Meereshochstände in Nordwest-Europa als Bezugsflächen für tektonische und isostatische Bewegungen
}

\author{
Von Paul Wolds te d t. Mit 3 Abb. im Text
}

Der Gedanke der glazial-eustatisch bedingten wärmezeitlichen Hochstände des Weltmeeres hat in den letzten Jahrzehnten weitgehende Anerkennung gefunden. Neuerdings hat sich vor allem F. ZEUner (1945) mit ihnen beschäftigt. Als weit verbreitete und in ihrem Alter gut bestimmte Niveaus können folgende gelten:

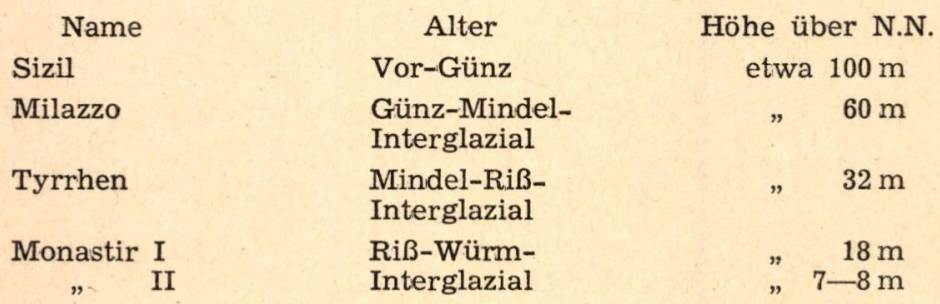

Von dem noch älteren Calabrischen Niveau sei hier abgesehen, da seine Stellung in diesem System noch umstritten ist.

Die genannten Ozeanspiegel sind also Meereshochstände in Warmzeiten, in denen alles oder wenigstens der größte Teil des Eises auf der Erdoberfläche geschmolzen und dem Weltmeer zugeführt war. Während der Eiszeiten war ein Teil des Ozeanwassers in Form von großen Inlandeisen festgelegt. Der Ozeanspiegel muß dementsprechend in den Eiszeiten wesentlich tiefer gelegen haben. Theoretische Berechnung (u. a. E. Antevs 1928, R. F. Flint 1947) und tatsächliche Beobachtung (u. a. A. C. Blanc 1942, M. Pfannenstiel 1944, 1949) kommen für die letzteiszeitliche Absenkung des Ozeanspiegels auf $90-100 \mathrm{~m}$. In den vorhergehenden größeren Vereisungen (Mindel und Riß) muß die relative Absenkung des Ozeanspiegels noch stärker gewesen sein. Man hat bis $200 \mathrm{~m}$ angenommen, ein Betrag, der jedoch reichlich hoch erscheint und rein glazialeustatisch kaum erklärt werden kann. Versucht man nun, das Bild des fallenden und steigenden Ozeanspiegels während der Glazial- und Interglazialzeiten schematisch in einer Kurve darzustellen, so ergibt sich das in Abb. 1 dargestellte Bild. Es muß besonders hervorgehoben werden, daß es sich hier um ein ganz rohes Schema handelt, das eine bewußte Vereinfachung der in Wirklichkeit viel komplizierteren Dinge bedeutet.

Was am meisten auffällt und nicht ohne weiteres zu erklären zu sein scheint, ist das immer tiefere Sinken des Meeresspiegels von einer Interglazialzeit zur nächsten (vgl. hierzu auch Abb. 76 in ZEUNER 1945). Man könnte zunächst daran denken, daß bei den späteren Vereisungen immer mehr Wasser in Form von Eis festgelegt und in den folgenden Interglazialzeiten ein immer mehr zunehmender Anteil nicht mehr abgeschmolzen sei. Das würde bedeuten, daß die Interglazialzeiten, je jünger sie sind, um so kühler sein müßten. Unsere Beobachtungen bestätigen dies jedoch nicht. Sowohl die Mindel-Riß- wie die Riß-Würm-Interglazialzeit scheinen in ihrem Optimum nicht wesentlich über das Klima-Opti- 


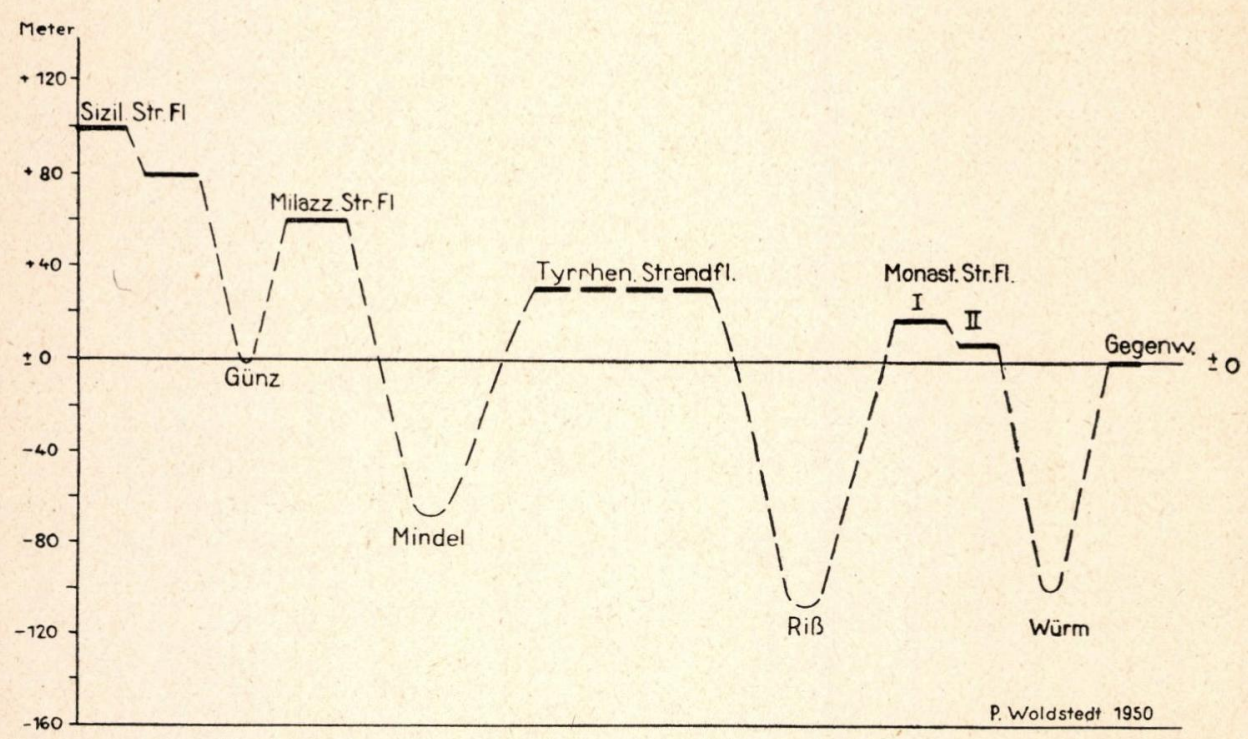

Abb. 1 Sroßschwankungen des Meeresspiegels im Laufe des Quartärs (stark schematisiert).

mum der Nacheiszeit, d. h. der gegenwärtigen Interglazialzeit, hinausgegangen zu sein.

Aber auch eine Rechnung, wie sie Antevs (1920), Ramsay (1930), Penck (1933) und DALY (1934) durchgeführt haben, ergibt, daß das Schmelzen des gesamten jetzt noch vorhandenen Eisvorrates auf der Erde (besonders von der Antarktis und Grönland) den Ozeanspiegel um höchstens 40-60 m ansteigen lassen würde. Die neueste Schätzung, die R. F. Flint (1947, S. 431) vorgenommen hat, kommt sogar nur zu einem Ansteigen des Meeresspiegels um $24 \mathrm{~m}$, ein Betrag, der allerdings reichlich niedrig erscheint. Aber nehmen wir selbst die höchste Schätzung von $60 \mathrm{~m}$ als richtig an, so würde damit gerade der Stand des Milazzo erreicht werden. Das Sizilische Niveau kann damit nicht erklärt werden, noch weniger das Kalabrische und die noch höheren jungtertiären. Es müssen also andere Ursachen vorhanden sein für dies kontinuierliche Absteigen des Ozeanspiegels, Ursachen, die vielleicht mit einer jungen Vertiefung der Ozeanböden zusammenhängen könnten (vgl. u. a. UMBgrove 1939, Baulig 1935, 1948).

Ein weiterer Punkt, der einer näheren Erläuterung bedarf, ist die Frage, ob wir überhaupt mit - geologisch gesprochen - langdauernden gleichbleibenden Meeresspiegeln rechnen dürfen. Findet, unabhängig von den glazial-eustatischen Bewegungen, eine mehr oder weniger gleichmäßig verlaufende Senkung des Ozeanspiegels im Laufe von Jung-Tertiär und Quartär statt - und damit müssen wir anscheinend rechnen - so können wir in den Interglazialzeiten nicht e in en langdauernden Spiegelstand erwarten, sondern einen allmählich absinkenden ${ }^{\mathbf{1}}$ ).

Aber dieses Absinken ging wahrscheinlich in Etappen, d.h. ruckweise, vor sich. In der letzten Interglazialzeit sind bisher zwei Hauptspiegelstände nachgewiesen, und zwar in etwa $18 \mathrm{~m}$ und $7-8 \mathrm{~m}+$ N.N.. Es ist nicht nötig anzuneh-

1) Diese Ansicht wurde auch von R. Grahmann in einer Diskussion bei Gelegenheit der Kölner Tagung der Geologischen Vereinigung am 7. 1. 51 geäußert. 
men, daß der zweite tiefere Meeresspiegel nur dadurch zustande gekommen sei, $\mathrm{da} \beta$ in einem kühleren, späteren Abschnitt der letzten Interglazialzeit schon wesentlich mehr Wasser in Form von Eis festgelegt gewesen sei. Die tiefere Lage könnte auch mit dem allgemeinen Absinken des Meeresspiegels zusammenhängen.

$\mathrm{Daß}$ ausgeprägte, zu bestimmten Meeresständen gehörige Strandflächen in verhältnismäßig kurzer Zeit entstehen können, haben die eingehenden Untersuchungen der schwedischen und finnischen Forscher im Gebiet der spät- und nacheiszeitlichen Ostsee gezeigt. Wir müssen annehmen, daß im Laufe des Quartärs eine Unzahl von einzelnen Strandflächen gebildet worden ist, von denen aber nur wenige erhalten geblieben sind. Die wichtigsten können wir, wie schon gesagt wurde, in die Hauptinterglazialzeiten einordnen.

Gelingt es nun, einen für einen ganz bestimmten Abschnitt des Quartärs charakteristischen Meeresspiegel über ein größeres Gebiet zu verfolgen, so haben wir damit ein Mittel an der Hand, um spätere Verbiegungen festzustellen, seien diese nun echt tektonischer oder isostatischer Art.

Wir versuchen dies zunächst mit dem Spiegel des letztinterglazialen EemMeeres in seiner optimalen Phase, und zwar auf einem Profil, dessen Lage aus Abb. 2 hervorgeht. Dieser Spiegel läßt sich allerdings in Schleswig-Holstein im Eidergebiet nur ungefähr festlegen. Nach freundlicher brieflicher Mitteilung von E. Dittmer-Husum (vgl. auch Dittmer 1941) liegen die höchsten Punkte von anstehendem, nicht gestörtem Eem bei $6-7 \mathrm{~m}-\mathrm{NN}$. G. v. d. Brelie gibt für die höchsten brackischen Bildungen $4-5 \mathrm{~m}-\mathrm{NN}$ an (briefl. Mitt.; vgl. auch G. v. d. Brelie 1951). Man kann danach den Spiegel des Eem-Meeres zu wenige Meter unter NN annehmen. Das ist natürlich nur ein angenäherter Wert, der aber vorläufig genügen kann.

Einen zweiten Punkt, an dem wir die Oberfläche des Eem-Meeres einigermaßen festlegen können, finden wir in der Gelderschen Vallei südlich der Zuider

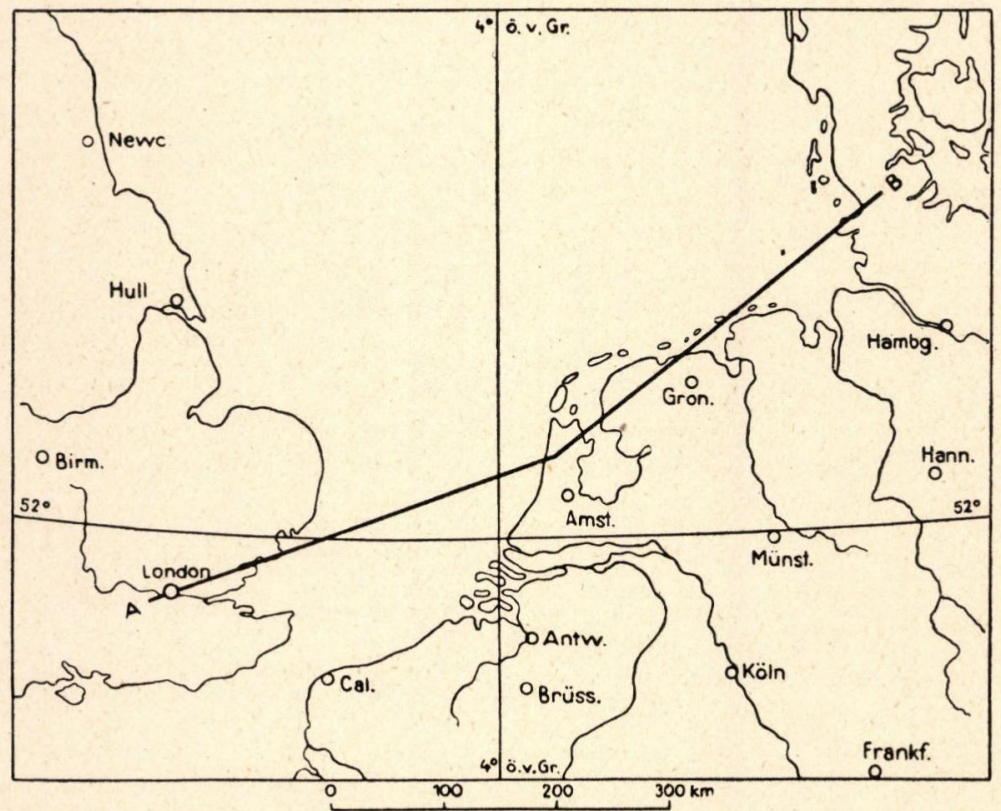

Abb. 2. Lage des Profils von Abb. 3. 
See. Hier können wir sie zu etwa $10 \mathrm{~m}-\mathrm{NN}$ annehmen. Weiter westlich scheint sie jedoch noch tiefer zu liegen. Pannexoek \& Reinhold (1949) geben die Oberfläche der Eemschichten weiter westlich, an der Küste, zu etwa $20 \mathrm{~m}-\mathrm{AP}$. an.

Wir versuchen schließlich, den Spiegel des Eem-Meeres im südlichen England, d. h. im unteren Themsegebiet, zu ermitteln. Unmittelbare Ablagerungen des Eem-Meeres sind dort nicht vorhanden. Dagegen finden wir nach den Ausführungen von F. ZEUNER (1945) im Themse-Mündungsgebiet zwei Terrassenaufschotterungen, die in der letzten Interglazialzeit gebildet sein müssen. Eine höhere, die Taplow-Terrasse, ist nach ZEunER auf einen Meeresspiegel von etwa $18 \mathrm{~m}+\mathrm{NN}$ eingestellt. Entstehung und Zeitstellung der Taplow-Terrasse sind jedoch noch umstritten (vgl. dazu auch P. WoldstedT 1950).

Eine jüngere Terrasse, die Obere Floodplain-Terrasse, ist auf einen Meeresspiegel von etwa $7-8 \mathrm{~m}+\mathrm{NN}$ eingestellt. Die Aufschüttung der höheren Teile dieser Terrasse muß in einer ausgeprägten Warmzeit erfolgt sein. Denn die Ablagerungen enthalten nach Zeuner (1945, S."126) u. a. Reste von Hippopotamus und Elephas antiquus. Das deutet auf eine Aufschüttung im klimatischen Optimum der letzten Interglazialzeit. In dies Optimum gehört aber auch das EemMeer. Man kann also mit einiger Wahrscheinlichkeit den Spiegel des EemMeeres im Themse-Mündungsgebiet auf etwa 7-8 $\mathrm{m}+\mathrm{NN}$ ansetzen.

In unser Profil, Abb. 3, haben wir die eben verfolgte Fläche als Riß-WürmMeeresspiegel („Monastir“) eingetragen, ohne an dieser Stelle genauer auf die Frage einzugehen, ob wir in der letzten Interglazialzeit zwei ausgeprägte Stände des Weltmeeres („Monastir I“ und „M. II“) hatten.

Wir versuchen nun, in ähnlicher Weise den Spiegel der Holsteinsee, d. h. des Meeresspiegels in der Mitte der Mindel-Riß-Interglazialzeit, den wir als „Tyrrhen" bezeichnen, festzulegen. Durch einen glücklichen Zufall gelingt dies annäherungsweise in denselben Gebieten, die wir eben herangezogen haben. Im westlichen Schleswig, und zwar auf Sylt, liegt nach freundlicher Mitteilung von E. Dittmer die Oberkante der anscheinend nicht gestauchten Tonvorkommen der Holstein-See bei etwa $3 \mathrm{~m}-\mathrm{NN}$. Von Ditrmer (1939) ist weiter der sog. „Husumer Sand" als eine strandnahe Ablagerung der Holstein-See nachgewiesen worden. Nach Lage der Dinge kann die Oberfläche der Holstein-See in diesem Gebiet annäherungsweise bei etwa NN angenommen werden. Sie ist also in diesem Gebiet vielleicht um ein Geringes höher anzusetzen als der Spiegel des Eem-Meeres.

Wir finden die Ablagerungen der Holstein-See wieder im nördlichen Holland (Westfriesland). Nach P. Tesch (1942) und A. Brouwer (1949) wird die Oberkante ihrer Ablagerungen hier in etwa $30-55 \mathrm{~m}-$ A.P. angetroffen. Für die Darstellung auf unserm Profil nehmen,wir den Wert von $30 \mathrm{~m}$-A.P. Die Oberkante derselben Schicht liegt im Rheinmündungsgebiet bei etwa $22-25 \mathrm{~m}-\mathrm{A} . P$. Im östlichen England schließlich haben wir als Äquivalent der Holsteinsee wahrscheinlich einen Teil der Corton Beds an der Ostküste von East Anglia, weiter auch das Vorkommen von Kirmington südlich der Humber-Mündung anzusehen. Das Vorkommen von Kirmington ist die Bildung eines Flußästuars, die auf einen damaligen Meeresspiegel in rund $30 \mathrm{~m}+\mathrm{NN}$ eingestellt ist. Die Oberkante der Corton Beds weist auf einen ähnlichen Meeresspiegel hin.

Schließlich können wir wieder im unteren Themsegebiet Anhaltspunkte für den Meeresspiegel im Optimum der Mindel-Riß-Interglazialzeit gewinnen. Die Oberkante der warmen Aufschüttungen der Boyn-Hill-Terrasse ist nach F. ZEUNER (1945, S. 124) eingestellt auf ein Meeresniveau von 100 Fuß über O.D., d. h. 
auf wenig mehr als $30 \mathrm{~m}+\mathrm{NN}$. Das aber ist die normale Höhe des Tyrrhens, wie sie vor allem in Nordfrankeich und im Mittelmeer festgestellt ist. Im südlchen England liegt also die Tyrrhen-Fläche heute um etwa $60 \mathrm{~m}$ höher als in Nordholland.

Wenn wir nun versuchen wollen, noch ältere Strandflächen in ähnlicher Weise zu verfolgen, so ergeben sich dabei etwas größere Schwierigkeiten. Am leichtesten ist dies wieder im unteren Themsegebiet möglich. Wie vor allem WooldRIDGE (1928) dargelegt hat, spricht eine in etwa $200 \mathrm{Fuß}$ Höhe gelegene Ebenheit des Gebietes, die sich dort vor der ersten nordischen Eisinvasion ausgebildet hatte, für einen Meeresspiegel in rund $60 \mathrm{~m}+\mathrm{NN}$ während dieser Zeit. Das aber war der Milazzo-Meeresspiegel, der in die Günz-Mindel-Interglazialzeit gestellt wird. Auf diesen Meeresspiegel ist die sog. Winter-Hill-Terrasse, bzw. ihr von Zeuner (1945, S. 119) als Finchley-Leaf bezeichneter Teil, eingestellt. Wie in den beiden früher untersuchten Fällen finden wir also im unteren Themsegebiet eine normale, in ihrer Höhe kaum veränderte Lage des alten Meeresspiegels vor.

Schwieriger liegen die Dinge in Holland. Die mächtigen fluviatilen Aufschüttungen, die in den nordwestlichen Niederlanden bis in $200 \mathrm{~m}$ Tiefe liegen, werden heute von den niederländischen Geologen zum Teil der Mindeleiszeit, zum Teil aber auch der Mindel-Riß-Interglazialzeit und der Riß-Eiszeit zugeschrieben (abgesehen von noch jüngeren Bildungen).

Unter den fluviatilen Schichten, die bei Alkmaar bis auf etwa $228 \mathrm{~m}$-A.P. heruntergehen (vgl. A. J. PANnEKoek \& Th. Reinhold 1949), folgt das marine Altpleistozän. Wir können für dies Gebiet mit einiger Wahrscheinlichkeit ein günzmindel-interglaziales Alter der höchsten marinen Bildungen annehmen. Es spricht manches dafür, daß die Regression, die nach der Bildung dieser höchsten marinen Schichten einsetzte, mit der beginnenden Mindeleiszeit zusammenhing. Wir können also die Milazzofläche in diesem Gebiet zu rund $225 \mathrm{~m}$-A.P. annehmen, d. h. etwa $285 \mathrm{~m}$ tiefer als im unteren Themsegebiet.

$\mathrm{Daß}$ die Milazzofläche sich zwischen dem unteren Themsegebiet und Nordholland herunterbiegt, das geht auch aus einer anderen Beobachtung hervor. Wenig nördlich der Verbindungslinie zwischen dem unteren Themsegebiet und Nordholland liegt an der Nordküste von East Anglia das berühmte Cromer Forest Bed, das allgemein in die Günz-Mindel-Interglazialzeit gestellt wird. Seine in einem Flußästuar abgelagerten Schichten tretèn hier annähernd im jetzigen Meeresniveau auf. Da sie seinerzeit auch etwa im Meeresniveau gebildet sein müssen, das aber damals rund $60 \mathrm{~m}$ höher lag, beträgt die Absenkung der Milazzofläche zwischen London und der Küste von Norfolk, d. h. auf knapp $200 \mathrm{~km}$, rund $60 \mathrm{~m}$. Viel stärker aber ist die Absenkung der Milazzofläche zwischen Cromer und Nordholland, die auf eine Entfernung von rund $225 \mathrm{~km}$ etwa $225 \mathrm{~m}$ beträgt.

Die Milazzofläche im westlichen Schleswig zu rekonstruieren, gelingt nur annähernd. In Sylt wissen wir durch die Untersuchungen von WirTz \& Illies (1951), daß wahrscheinlich während der Günzeiszeit das Meer hier nach Westen zurückwich. Von einer neuen Transgression in der Günz-Mindel-Warmzeit sind hier keine Spuren vorhanden. Die Bildungen, die wir vielleicht in die Warmzeit zu stellen haben, sind terrestrischer Art. In welcher Höhe wir den MilazzoSpiegel zu suchen haben, muß daher offen bleiben. Wir haben ihn am ehesten etwa in der Höhe des jetzigen Meeresspiegèls anzunehmen. 
Ganz unsicher werden unsere Angaben, wenn wir schließlich versuchen, noch die Sizil-Fläche zu rekonstruieren. Wir wollen aber trotz alledem den Versuch machen, wenn er auch stark hypothetisch ist. Für das untere Themsegebiet hat WoOLDRIDge (1927; vgl. auch ZEuner 1945, S. 117) es wahrscheinlich gemacht, daß die 400 Fuß-Ebenheit des Gebietes zum Sizil gehört. Wir dürfen also vielleicht annehmen, daß der Spiegel des Sizil-Meeres im Londoner Gebiet damals bei rund $100-120 \mathrm{~m}$ gelegen habe.

Die Frage, wo etwa im nördlichen Holland heute die Sizil-Fläche zu suchen sei, ist außerordentlich schwierig zu beantworten. Wir können sie - allerdings mit sehr großer Unsicherheit - irgendwo im marinen Altpleistozän suchen, wo aber im besonderen, das entzieht sich einstweilen unserer Beurteilung. Immerhin dürfte soviel feststehen, daß wir sie im nördlichen Holland un ter dem Milazzo zu suchen haben. Die Abbiegung der Sizil-Fläche vom Themsegebiet nach Nordholland muß also noch stärker gewesen sein als die der Milazzofläche.

In Schleswig ist es bisher nicht möglich, das Niveau anzugeben, in dem dort etwa die Sizil-Fläche zu suchen ist. Aber man wird hier vielleicht annehmen können, daß sie über dem jetzigen Meeresspiegel liegt, wenn auch vielleicht nicht sehr hoch.

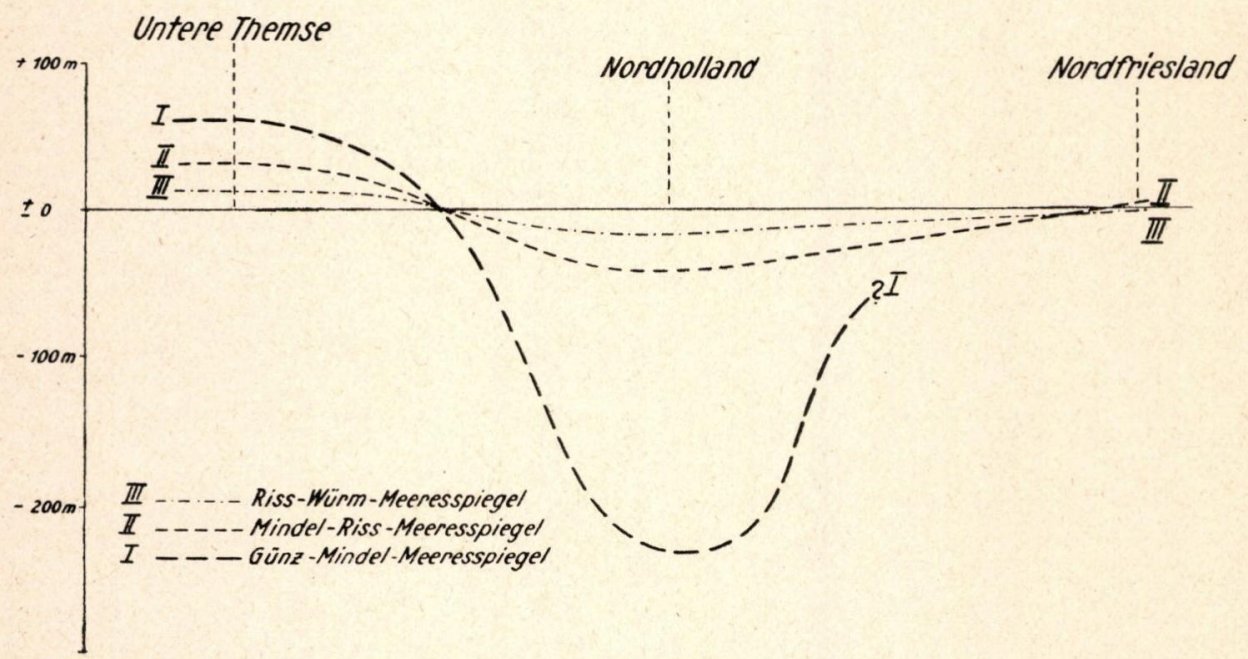

Abb. 3. Heutige Lage der alten, interglazialen Meeresspiegel zwischen Themsegebiet und Nordfriesland (der Meeresspiegel II ist in Nordfriesland etwas zu hoch eingezeichnet worden).

In Abb. 3 sind nun die Flächen des Milazzo, Tyrrhen und Monastir in Form eines Profils vom unteren Themsegebiet über Nordholland nach Westschleswig zusammengestellt. Vom Sizil ist abgesehen worden, weil die Unsicherheiten bei seiner Rekonstruktion zu groß sind.

Die Betrachtung des Profils zeigt, daß die Flächen im Londoner Gebiet normal untereinander liegen, d. h. die älteste oben, die jüngste unten. In Nordholland liegen sie genau umgekehrt, die älteste ganz unten, dann folgen, näher zusammen, die mittlere und die obere Fläche. In Schleswig schließlich liegen sie wahrscheinlich alle drei nahe beieinander.

Gehen wir davon aus, daß das Londoner Gebiet seine ursprüngliche Höhenlage beibehalten habe - gegenüber dem Meeresspiegel ist es relativ gestiegen, 
denn dieser ist ja seit dem Milazzo um rund $60 \mathrm{~m}$ gesunken -, so ist Nordholland seit dem Milazzo um etwa $285 \mathrm{~m}$ gesunken. Der Hauptbetrag dieser Senkung hat stattgefunden zwischen dem Milazzo und dem Tyrrhen. In dieser Zeit, d.h. im wesentlichen in der Mindelzeit, ist Nordholland gegenüber dem Themsegebiet um 190-200 m gesunken.

Bei dieser Absenkung handelt es sich offenbar um echte tektonische Bewegungen. Nach den Beobachtungen im deutschen Niederrheingebiet und in den südöstlichen Niederlanden (vgl. für letzteres bes. J. T. S. ZonNEveld 1947) durchsetzen Grabenbrüche und ähnliche tektonische Formen dies Gebiet in nordwestlicher Richtung. Ihre weitere Fortsetzung nach Nordwesten und Norden kennen wir nicht, da wir hier auf Bohrungen angewiesen sind. Es ist aber ein ähnlicher tektonischer Bau anzunehmen. Von diesem Gesichtspunkt aus müßte man im Profil vielleicht nicht eine glatte Kurve, sondern Staffelbrüche zeichnen. Ob man aber den ganzen Betrag rein tektonisch zu deuten hat, erscheint fraglich. Wir müssen in diesem Gebiet vielleicht auch mit einer isostatischen Einbiegung der Erdkruste unter der Last der hier sich anhäufenden Sedimente rechnen.

Wie schon gesagt wurde, muß die Hauptabsenkung zwischen der Bildung der Milazzo- und der Tyrrhen-Fläche erfolgt sein, d.h. grob gesprochen, in der Mindelzeit. Aber später müssen diese Bewegungen in demselben Sinne, jedoch abgeschwächt, weitergegangen sein. Wir können den Betrag jeweils aus unserem Profil ablesen.

Ganz anders aber liegen die Dinge in Schleswig. Wenn wir annehmen, daß dort die Milazzofläche etwa in der Höhe des heutigen Meeresspiegels liegt, so würde das gegenüber dem Themsegebiet eine Absenkung von rund $60 \mathrm{~m}$ bedeuten. Die Tyrrhenfläche ist in Schleswig gegenüber dem Tyrrhen im Themsegebiet um etwa $30 \mathrm{~m}$ gesunken. Die entsprechende Zahl für das Monastir mag etwa $10-12 \mathrm{~m}$ betragen.

$\mathrm{Ob}$ wir in diesem Falle ebenfalls mit einer tektonischen Senkung zu rechnen haben, läßt sich vorläufig nicht sagen. Man wird jedenfalls auch daran denken müssen, daß in diesen Gebieten eine isostatische Absenkung stattfand unter der Last der sich hier anhäufenden glazialen Sedimente.

Es sei zum Schluß nochmals betont, daß unsere Ableitungen noch mit starken Unsicherheiten behaftet sind. Diese liegen vor allem darin, daß der strikte Nachweis einer absoluten Gleichaltrigkeit der Meersstände meist sehr schwierig ist. Weiter bleibt bei der Bestimmung des jeweiligen Meeresspiegels oft noch eine beträchtliche Unsicherheit. Es muß das Ziel sein, diese Werte weiter zu verfeinern und für möglichst viele Punkte einwandfrei zu bestimmen. Wir werden dann die Möglichkeit haben, die Abweichungen der jetzigen Lage dieser Flächen von der theoretisch $\mathrm{zu}$ erwartenden festzustellen und die Bewegungen, die $z u$ der veränderten Lage geführt haben, sowohl nach ihrem Ausmaß wie nach ihrer zeitlichen Einstufung genauer festzulegen. Untersuchungen dieser Art sind wichtig für die sog. "Senkungsfrage“ an der deutschen Nordseeküste. Sie zeigen uns die langfristigen Bewegungstendenzen der einzelnen Räume und gestatten uns vielleicht auch, besondere tektonische Anteile herauszuschälen, die möglicherweise für einzelne Gebiete in Frage kommen.

$\mathrm{Schriftennach}$ weis

Antevs, Ernst: The last glaciation. - American Geograph. Society, Research Series Nr. 17, New York 1928. - Quaternary marine terraces in non glaciated regions and changes of level of sea and land. - Amer. Journ. of Sci. 17, S. $35-49.1929$. 
Baulig, H.: The Changing Sea Level. - The Instit. of British Geographers, Publ. Nr. 3. London 1935. - Problèmes des terrasses. - Un. géogr. internat. ,6. Rapport Commiss. des Terrasses. 109 S. Louvain 1948.

Blanc, A. C.: Variazioni climatische ed oscillazioni della linea de riva nel Mediterraneo centrale durante l'Era glaciale. - Geologie d. Meere u. Binnengewässer 5, S. 137-219. 1942.

v. d. Brelie, G.: Die junginterglazialen Ablagerungen im Gebiet des Nord-OstseeKanals. - Schr. natw. Ver. Schlesw.-Holst. 25, S. 100-107. Kiel 1951.

Brouwer, A.: Pollenanalytisch en geologisch onderzoek van het Onder- en MiddenPleistoceen van Noord-Nederland. - Leidse Geol. Medel. 14 B, S. 259-346. 1949.

Daly, A. R.: The Changing World of the Jce Age. - New Haven, Yale University Press. 1934.

DitTMER, Ernst: Zur Verbreitung altinterglazialer Meeresablagerungen in Nordfriesland. - Westküste 2, S. 123-132. 1939. - Das nordfriesische Eem. - „Kieler Meeresforschungen“ S. 169-199. 1941.

Flint, R. F.: Glacial Geology and the Pleistocene Epoch. - New York und London 1947.

Pannekoek, A. J. \& Reinhold, Th.: Een geologisch profil langs de Nederlandse Kust. Mededeel. Geol. Stichting N.S. 3, S. 69-74. 1949.

PENCK, Albrecht: Eustatische Bewegungen des Meeresspiegels während der Eiszeit. Geogr. Z. 39, S. 329-339. 1933.

Pfannenstiel, Max: Die diluvialen Entwicklungsstadien und die Urgeschichte von Dardanellen, Marmarameer und Bosporus. - Geol. Rundschau 34, S. 342-434. 1944. - Klimatisch bedingte Spiegelschwankungen des Mittelmeeres im Quartär und die paläolithischen Kulturen. - Mitt. geol. Ges. Wien 36-38, S. 257-263. 1949.

Ramsay, W.: Changes of sea-level resulting from the increase and decrease of glaciations. - Fennia 52, Nr. 5. 1930.

Tesch, P.: De Noordzee van historisch-geologisch standpunt. - Med. Geol. Stichting, Ser. A, Nr. 9. 1942.

Umвrove, J.H. F.: On Rhythms in the History of the Earth. - Geol. Mag. 76, S. 116-129 London 1939.

Wirtz, D. \& Illies, H.: Plio-Pleistozängrenze und Günzeiszeit in Nordwestdeutschland. - Eiszeitalter u. Gegenwart 1, S. 73-83. 1951.

Woldstedt, Paul: Das Vereisungsgebiet der Britischen Inseln und seine Beziehungen zum festländischen Pleistozän. - Geol. Jahrb. 65, S. 621-640. Hannover 1950.

Wooldridge, S. W.: The Pliocene History of the London Basin. - Proc. Geol. Assoc. London 38, S. 49-132. 1927. - The 200-foot Platform in the London Basin. Proc. Geol. Ass. London 39, S. 1-26. 1928.

Zeuner, Frederick, E.: The Pleistocene Period. Its Clinate, Chronology and Faunal Succession. - Ray Society, Vol. 130. London 1945.

Zonneveld, J. I. S.: Het Kwartair van het Peelgebied en de naaste omgeving. - Med. Geol. Sticht. Ser. C VI. 3. 1947.

Ms. eing.: Mai 1951.

\section{Zur Theorie der Gletschererosion in Tälern}

Von H. Lou is, Köln. Mit $3 \mathrm{Abb}$.

\section{Erosionstheorie und Theorie der Gletscherbewegung}

Im Gegensatz zu den, vom Relief des Untergrundes weitgehend unabhängigen Erscheinungen übergeordneter Vergletscherung, wie sie als Plateauvergletscherung oder als Inlandeis entgegentreten, passen sich wie bekannt, beim Typus der sogenannten untergeordneten Vergletscherung Gletscher einem schon vorher ausgebildeten Tälerrelief ein. Die Eisbewegung wird hierbei durch die Täler dirigiert, wirkt sich andererseits in einer mehr oder weniger kräftigen Umformung der betroffenen Täler aus, so daß diese nach Schwinden des Eises von nicht vergletschert gewesenen Tälern deutlich zu unterscheiden sind. 